\title{
Dishing up the ocean's riches
}

\author{
Orla Smith
}

"The richness of the sea is in its genetic resources and we must look to biotechnology to preserve this genetic diversity," said Rita Colwell, president of the University of Maryland Biotechnology Institute (College Park, MD), presenting the plenary address at a recent meeting on marine biotechnology*. The scope of the meeting, which was sponsored by Sea Grant-a partnership of academia, government, and industry that supports university-based marine science researchechoed her sentiments, with speakers discussing topics as diverse as the aquaculture of edible fish species, genetic engineering of fish stock, fish vaccine design, the development of biodegradable polymers from marine organism proteins, and bioremediation.

The first speaker, Perry Hackett of the University of Minnesota in St. Paul, began by discussing the difficulties he encountered when he first attempted to genetically engineer bigger fish. These difficulties convinced him of the necessity for designing better strategies for integrating and expressing foreign DNA in fish chromosomes. "A major problem with making transgenic [fish] is that the expression of a transgene can be modified according to where it ends up in the genome," says Hackett. To reduce these "position effects," his group designed chloramphenicol acetyltransferase (CAT) reporter gene constructs in which the transgene is flanked by either fruit fly or chicken border elements-these elements act as insulators against interference from neighboring chromatin. According to Hackett, mature zebrafish showed uniform and reproducible CAT expression following injection of the construct into one-cell embryos. Now he is redesigning the construct to include a retroviral integrase that should increase the efficiency of transgene integration into chromosomes still further.

Hackett's team has also identified mobile DNA elements (transposons) in salmon that are similar to the $\mathrm{Tcl}$ transposons of Caenorhabditis elegans. Using synthetic salmonid Tcl-like DNA elements in zebrafish embryos, they detected movement of externally added antibiotic resistance genes. Their work demonstrates the potential of synthetic transposons as vectors for efficient delivery of transgenes and in insertional mutagenesis and gene mapping.

In another talk, Jo-Ann Leong of Oregon State University discussed ongoing work

Orla Smith is assistant editor, News and Views, Nature Medicine, Washington, DC. developing vaccines to prevent the spread of microbial disease in farm-reared fish-infections that cost the aquaculture industry "millions of dollars each year." According to Leong, "Infectious hematopoietic necrosis virus (IHNV) alone kills between 2 and 20 million trout and salmon and there is no effective vaccine." For the past decade, she has been

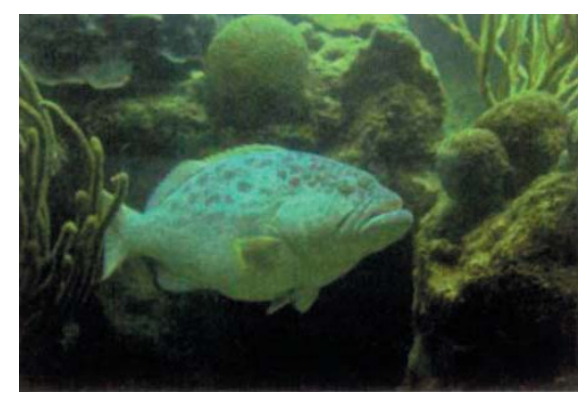

Figure 1. More than $70 \%$ of the earth is covered by ocean-a rich source of food, biochemicals, and pharmaceuticals.

designing vaccines to protect farm-reared and native fish against two fatal viruses: $1 H N V$ and infectious pancreatic necrosis virus (IPNV).

Field trials in which fish fry were bathed in concentrated IHNV subunit glycoprotein vaccine for four minutes demonstrated that $98 \%$ of the fish exposed to infectious challenge were protected. When the glycoprotein was produced in high-density fermentation vats, however, the vaccine conferred only variable protection. This prompted Leong and her graduate student, Eric Anderson, to switch their attention to DNA vaccines. They found that injection of a DNA construct of the IHNV glycoprotein gene under control of a CMV promoter into the skeletal muscle of trout fry elicited sufficient antibody to provide protection against IHNV challenge. In an interesting twist to this work, Leong injected recombinant human interleukin (IL)-2 along with the IHNV DNA vaccine and obtained heightened protection against IHNV challenge in trout fry. "One of our long-term goals is to identify fish interleukins that may enhance the protective effects of vaccines," says Leong.

Another speaker at the conference highlighted the potential biotechnological value of one other of the ocean's residents-the common oyster. Hap Wheeler's early interest in mineralized tissue prompted him to study the composition of oyster shell-work that has "spawned" a biodegradable protein polymer with a wealth of unexpected applications. "It was the crystal growth inhibitory activity of polyaspartic acid (PAA) in the oyster shell protein that made us realize its application as a biodegradable, nontoxic water treatment agent for preventing crystal deposition in tanks and pipes," says Wheeler of Clemson University in South Carolina. Together with Larry Koskan at Donlar Corporation (Bedford Park, IL), he helped perfect a method of thermal polymerization to enable rapid synthesis of large quantities of PAA.

Water treatment is not the only application of these fascinating PAA polymers; they have also proved useful as dispersants in detergents and, perhaps most surprisingly, as enhancers of nutrient uptake in crop plants. "We think the protein polymer, in contact with the roots of crop plants, attracts valuable cations into the tiny root hairs," speculates Wheeler. Field trials have proven successful and PAA is now being marketed as a crop enhancer, with projected sales of $\$ 150$ million per year. Longer, modified PAA molecules have been found to absorb many times their weight in liquid. "We are also trying to produce PAA-based components for diapers that are degradable under compost conditions," says Wheeler. "The goal is to use these products to reduce the need for environmental remediation," he concludes.

Biodegradable diapers may solve some environmental problems, but effective bioremediation strategies are still required to rescue rivers and coastal waters contaminated with industrial toxic waste. In his talk, Ralph Portier from Louisiana State University, (Baton Rouge, LA) described the use of an immobilized microbe bioreactor using diatomaceous material extruded into beads as a biosorptive support for high-density populations of bacteria-the ultimate "bug condominium." A portable bioreactor containing immobilized Xanthobacter, at a density of $10^{7}-10^{11}$ cells/g of bead material, has proved very successful in removing the carcinogen ethylene dichloride (EDC) from contaminated groundwater. "With a retention time of 4.5 hours and a biodegradation rate of $>99 \%$, EDC-contaminated water can be processed at a rate of 360 liters per minute," says Portier. Portable Pseudomonas bioreactors have successfully recovered large sections of riverine and coastal areas contaminated with petroleum waste, reducing engineering costs in these areas by as much as $80 \%$. The latest bioreactor, supporting mixed colonies of Pseudomonas and Flayobacteria, removes contaminants such as creosote and other preservatives from salvaged wood, which can then be recycled.

"Sea Grant Marine Biotechnology Briefing" was held March 3, 1997 at the Renaissance Mayflower Hotel, Washington, DC. 